\section{RevistadePolítica Económica y Desarrollo Sostenible}

Centro Internacional de Política Económica para el Desarrollo Sostenible
Revista de Política Económica y Desarrollo Sostenible

EISSN: 2215-4167 • Vol. 2 (1) • Julio-Enero, 2016: 1-25

DOI: http://dx.doi.org/10.15359/peds.2-1.2

URL: http://www.revistas.una.ac.cr/politicaeconomica

Revista electrónica semestral publicada por el Centro Internacional de Política Económica para el Desarrollo Sostenible

Universidad Nacional, Lagunilla, Heredia Apartado 2393-3000

\title{
Creación de competencias y capacidades en el subsector lechero costarricense
}

\author{
Capability buildings and dairy sector in Costa Rica
}

Luis M. Barboza-Arias

\section{Resumen}

La dinámica productiva del subsector lechero en Costa Rica ha experimentado cambios importantes como consecuencia de su evolución histórica. Estas transformaciones propiciaron la incorporación de innovaciones que han surgido, en mayor o menor medida, como resultado de procesos institucionales de adaptación a las condiciones geográficas y climatológicas del país. Este estudio aborda la gestión de la innovación institucional en el subsector lechero costarricense y su objetivo principal es analizar los efectos sobre la creación de competencias y capacidades de productores lecheros. Se realiza una breve caracterización del subsector lechero, así como una descripción general de los actores organizacionales que participan en la promoción de mecanismos para la gestión de ideas y conocimientos. Se utiliza la metodología de estudios de caso, en particular, el análisis de tres experiencias que ejemplifican los efectos de la transferencia tecnológica en la organización del sector y la acción colectiva de productores primarios en localidades diferentes del país. El estudio concluye que el sistema nacional de innovación costarricense tiene un rol central en el establecimiento de la agenda de innovación agropecuaria y la transferencia tecnológica; sin embargo, presenta puntos de mejora en cuanto a apoyar los procesos de gestión del conocimiento y creación de capacidades. Se determina además la necesidad de continuar avanzando en el fortalecimiento de áreas estratégicas de acción conjunta que faciliten medios de participación efectiva a los ganaderos y un diálogo institucional coherente con las estrategias de desarrollo local inclusivo.

Palabras Clave: Capacidades humanas, Cooperativas, Desarrollo inclusivo, Innovación institucional, Lechería.

\begin{abstract}
Productive dynamics of the dairy sector in Costa Rica, underwent significant changes as a result of its historical evolution. Because of these changes, institutional actors at different levels of implementation promoted the incorporation of innovations, including adaptation processes to the geographical and climatic conditions of the country. This study focuses on the innovation management of the dairy sector in Costa Rica. Its main goal is to analyze the process of capability building of the dairy farmers, considering the importance of knowledge management initiatives. We use a case studies methology to introduce three specific experiences of innovation and technology transfer in different places of Costa Rica. Results suggest that National System of Innovation of country plays a special role in designing of strategic working agenda, however, key aspects could enhance if knowledge management and capability buildings approaches are considered. Finally, the paper concludes that the promoting of community participation mechanisms is important to build collective actions and setting up institutional dialogues for the inclusive local development strategies.
\end{abstract}

Key Words: Co-operatives, Dairy sector, Human capabilities, Inclusive development, institutional innovation. 
Revista de Política Económica • EISSN: 2215-4167

Vol. 2 (1) • Julio-Enero, 2016: 1-25

DOI: http://dx.doi.org/10.15359/peds.2-1.2

Barboza-Arias

URL: http://www.revistas.una.ac.cr/politicaeconomica

\section{Introducción}

En la última década, la actividad agropecuaria costarricense ha sido afectada de forma sensible por variables ambientales asociadas al cambio climático y transformaciones profundas en los términos de intercambio comercial internacional. En particular, el establecimiento de nuevos acuerdos comerciales con otras nacionales ha provocado un aumento en la importación al país de productos lácteos. Para el subsector lechero, esta situación se traduce en pérdida de competitividad de las fincas e industrias lácteas debido a las presiones sobre el precio de los productos locales, así como a rendimientos económicos menores provocados por una mayor competencia. Las organizaciones vinculadas al sector reconocen la necesidad de promover programas de apoyo con el fin de estimular innovaciones que permitan un mayor dinamismo de la producción y sirvan a la vez para mejorar la calidad de vida de los productores lecheros y sus familias. La estrategia principal ha sido la transferencia tecnológica de alto nivel.

La agenda de innovación y modernización técnica para el sector agropecuario establece líneas de acción puntuales para promover procesos de innovación a través de las diferentes organizaciones del subsector lácteo. Estas se caracterizan por el desarrollo de actividades de investigación y desarrollo (I+D) para el mejoramiento genético y nutricional del hato lechero. Sin embargo, a nivel local los productores a pequeña escala incorporan cambios y mejoras leves en su proceso productivo de manera rutinaria, que son, a su vez, resultado directo de prácticas empíricas y de la experiencia obtenida a partir del trabajo diario en la finca. Comprender la función que cumple el conocimiento tácito en los procesos de aprendizaje individual y colectivo de los productores lecheros representa una estrategia metodológica clave para analizar sus efectos en la creación de competencias y capacidades para la innovación, así como para valorar la viabilidad de las iniciativas de modernización que promueven el uso y apropiación de la tecnología como la estrategia principal de desarrollo.

Los casos de estudio que se presentan en este artículo: Cooperativa Dos Pinos R.L., Cooperativa Agroindustrial de Servicios Múltiples de Puriscal R.L. y Asociación de Productores Agropecuarios de Santa Cruz (Asoproa), constituyen ejemplos de que las cooperativas participan de forma activa en la gestión de ideas y conocimiento. Para los productores, el ambiente organizacional de estas asociaciones constituye un punto de encuentro e intercambio horizontal de información. Las cooperativas operan como "comunidades de prácticas", espacios semiformales donde los factores culturales y geográficos contribuyen a formar y caracterizan la naturaleza del vínculo de trabajo y los procesos de aprendizaje y capacitación de los productores.

\section{Sistemas nacionales de innovación: una aproximación teórico-conceptual}

Antes de continuar con el desarrollo del estudio, es importante clarificar el marco teóricoconceptual en el cual está sustentado. En primer lugar, resulta necesario definir qué se entiende por innovación. De acuerdo con Lundvall (1992), la innovación debe entenderse como el resultado de procesos continuos de aprendizaje, búsqueda y exploración. Van Dijk y Sandee (2002) señalan que la 
innovación es un componente central de la competitividad empresarial en el contexto de la economía global; para estos autores, la innovación se encuentra asociada al cambio tecnológico, que permite organizar el trabajo a partir de la introducción de nuevas técnicas de producción y el desarrollo de habilidades en los trabajadores. Edquist (1997, p.1) desarrolla una definición más amplia del concepto de innovación, relacionándolo con "las nuevas creaciones de significancia económica; las cuales pueden ser totalmente nuevas o nuevas combinaciones de elementos ya existentes".

Ahora bien, la innovación no es un acontecimiento repentino, que ocurre de forma aislada. El concepto de Sistema Nacional de Innovación permite identificar la existencia de instituciones que orientan - y en muchos casos definen- la gestión de la innovación en las empresas (Orozco, Ruiz y Corrales, 2015) y factores socioculturales que influyen sobre las dinámicas de desarrollo. La innovación no solamente puede darse a nivel de producto, también puede estar presente en el proceso productivo, la comercialización o la organización interna del trabajo; asimismo, puede ser radical, si se trata de algo por completo novedoso en el mercado, o incremental, cuando consiste en una adaptación de algo ya existente.

Arocena y Sutz (2002) desarrollan el concepto de "espacios interactivos de aprendizaje", espacios donde la innovación es el resultado de procesos de intercambio de conocimiento, guiados por organizaciones y personas a partir de las capacidades de aprendizaje. Lam (1998) plantea la importancia de distinguir entre distintos tipos de conocimiento y formas organizacionales, una estructura analítica que permite comprender las diferentes relaciones que pueden establecer actores e instituciones, y sus efectos sobre la capacidad de aprendizaje de los trabajadores.

Los aportes de Lam resultan de notable importancia para el presente estudio. Es fundamental entender el funcionamiento de "comunidades de prácticas" (Lam, 2002) en sectores como el lechero, donde el conocimiento es tácito, y en el cual los factores sociales como la edad de los productores y el nivel educativo tienen un efecto determinante sobre la creación de capacidades. Aunado ello, existe un conjunto de representaciones sociales que se integran a la cosmovisión que el productor tiene del paisaje local en que realiza su actividad productiva y contribuyen a modelar los aspectos subjetivos (como el arraigo, la pertenencia y el sentido), que determinan la relación del productor con su entorno y, en consecuencia, su proceso productivo.

\section{Diseño metodológico}

Para el desarrollo de este estudio se aplica una metodología basada en el diseño propuesto por Yin (1994) sobre estudios de caso. La principal fortaleza de esta metodología es que no se basa en análisis estadísticos, sino que permite tomar un grupo de estudios de caso y derivar generalizaciones analíticas que sean aplicables para el sector en su conjunto.

Se incluyen además algunos elementos metodológicos del Análisis de Múltiple Criterio para justificar la presencia de actores institucionales y académicos en el proceso de investigación. Para la obtención de la información y su posterior manejo, se realizaron entrevistas a empleados 
del sector público y consultores privados, así como visitas a productores lácteos de las localidades de Puriscal (San José) y Turrialba (Cartago).

\section{Breve contextualización del subsector lechero en Costa Rica}

\subsection{Dinámica del sector agropecuario}

La economía costarricense experimentó en las últimas décadas un desplazamiento de recursos y factores productivos hacia el sector terciario. En consecuencia, la tendencia de la actividad agropecuaria en el país ha sido la menor participación dentro de la producción nacional total. Para el año 1991, el sector agropecuario representó un 13\% del Producto Interno Bruto costarricense, mientras que para 2014 el porcentaje de participación fue de 9\% (Apéndice 1).

El sector pecuario, compuesto por los subsectores de ganadería de leche, ganadería de carne, avicultura, porcicultura y huevos, representó, en 2015, 30.20\% del valor agregado agropecuario (Gráfico 1).

\section{Gráfico 1.}

Participación de subsectores en el Valor Agregado Agropecuario, 2015

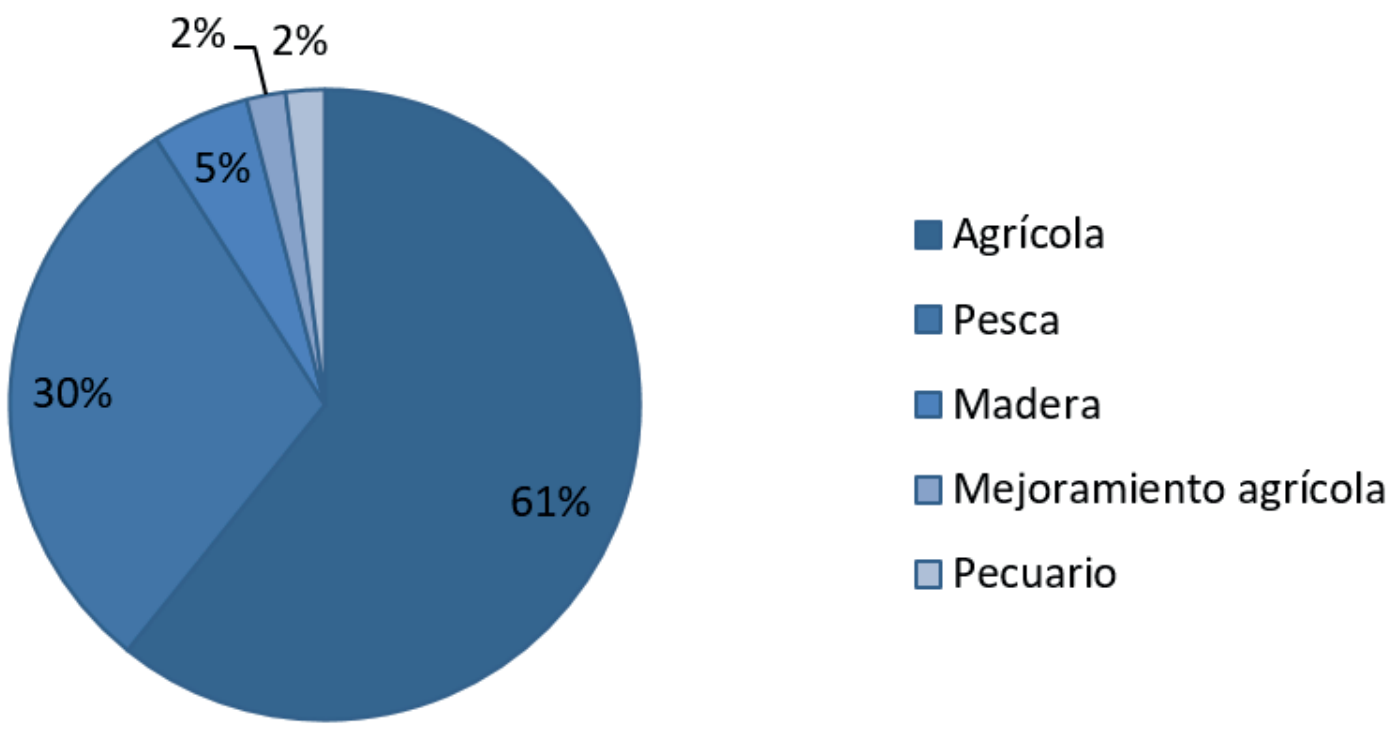

$\left.{ }^{*}\right)$ Porcentajes estimados para millones en colones corrientes.

Fuente: Elaboración propia con datos de Sepsa, 2016. 
En el Cuadro 1 se presenta la participación de cada uno de los subsectores productivos en el valor agregado del sector pecuario para los años 2012 al 2015.\}

Cuadro 1. Costa Rica: Valor agregado de las principales actividades del sector pecuario, 2012-2015, (millones de colones corrientes)

\begin{tabular}{lccccc}
\hline Actividades & $\mathbf{2 0 1 2}$ & $\mathbf{2 0 1 3}$ & $\mathbf{2 0 1 4}$ & $\mathbf{2 0 1 5}$ & $\begin{array}{c}\text { Participación } \\
\mathbf{2 0 1 5}\end{array}$ \\
\hline Lechería & 146670,75 & 144126,64 & 150399,76 & 156847,47 & $38.57 \%$ \\
\hline Pollos & 100580,84 & 104153,45 & 108274,13 & 111138,07 & $27.33 \%$ \\
\hline Ganado carne & 78644,44 & 90340,36 & 90113,15 & 91576,71 & $22.52 \%$ \\
\hline Huevos & 33007,88 & 32479,32 & 33305,66 & 37142,02 & $9.13 \%$ \\
\hline Porcinos & 7154,92 & 8801,93 & $9.515,48$ & $9.941,35$ & $2,44 \%$ \\
\hline Total & 366058,82 & 379901,69 & $391.608,18$ & $406.645,62$ & $100 \%$ \\
\hline
\end{tabular}

Fuente: Elaboración propia con datos del Ministerio de Agricultura y Ganadería, 2015

En términos porcentuales, dentro del sector pecuario la lechería contribuyó en el 2015 con casi un $40 \%$ del valor agregado y junto con la ganadería de carne aportaron con cerca de $60 \%$ del valor agregado pecuario. Un hecho importante es el ligero crecimiento de la actividad lechera en relación con el año 2014. De acuerdo con el Informe técnico de resultados del $1^{\circ}$ año de Gobierno, realizado por el Ministerio de Agricultura y Ganadería en el año 2015, este incremento estaba proyectado, dado el crecimiento del hato destinado a actividades de leche y doble propósito.

Los datos reflejan además que la actividad lechera continúa siendo una actividad agropecuaria importante, y este dinamismo, precisamente, puede favorecer la incorporación de innovaciones en el subsector. De acuerdo con datos de la Cámara Nacional de Productores de Leche (2015), la producción anual de leche del país alcanzó 1.077 millones de litros en el año 2014; es decir, casi tres millones de litros diarios. Según esta organización (2015), en su fase primaria la producción de leche representa un 9,9\% del valor agregado agropecuario, siendo superada en importancia relativa tan solo por la producción de piña y banano. El Gráfico 2 muestra la evolución del crecimiento de la producción de leche para el período 2010-21014. 


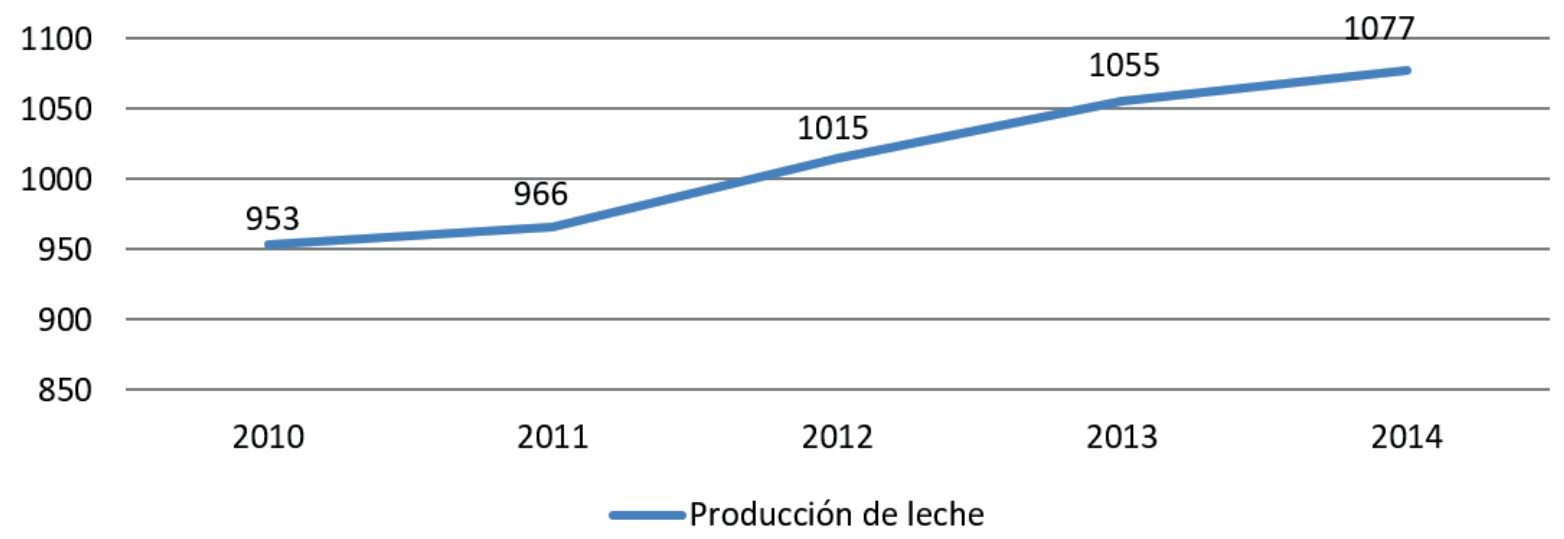

Gráfico 2. Costa Rica. Producción de leche 2010-2014 (en toneladas métricas)

Fuente: Cámara Nacional de Productores de Leche de Costa Rica, con datos del BCCR y Sepsa, 2015.

\section{Sub-sector lechero}

El aumento sostenido de la producción de leche en el país ha permitido, de acuerdo con la CNPL (2015), el ligero aumento del consumo nacional y las exportaciones de productos lácteos. Para el año 2014, las exportaciones de productos lácteos de Costa Rica ascendieron a 92.02 millones de kilos, con un valor total de 158.53 millones de dólares; esto representa un incremento de $20.6 \%$ en volumen y $17.64 \%$ en el valor de exportación en comparación con las cifras del año 2013 (Ministerio de Hacienda, 2015). Sin embargo, el precio por kilo exportado se vio disminuido en un $2 \%$ en relación con el año 2013, cayendo de US\$1.77 por kilo exportado en 2013 a US $\$ 1.72$ en 2014 (CNPL, 2015). El Gráfico 3 muestra el incremento del volumen y valor de exportación para el período histórico 2011-2014.

Por otra parte, el incremento del volumen exportado de productos lácteos se traduce también en una mayor dependencia del mercado, especialmente de los insumos necesarios para la producción. De acuerdo con Sergio Abarca, investigador del Instituto Nacional de Innovación y Transferencia en Tecnología Agropecuaria (Inta), el costo de la alimentación del ganado y la mano de obra aumenta a medida que la tecnificación del proceso productivo gana terreno y la calidad de la leche es mejor. Para Abarca (2015), existe una problemática grave con el precio de los granos como alimento bovino, el uso de los concentrados y la desgravación arancelaria estipulada por el Tratado de Libre Comercio entre Estados Unidos, Centroamérica y República Dominicana. En muchos casos, si bien existe un mal manejo de la parte nutricional y de alimentación de los animales en las fincas, los registros para la importación de materias primas y el exceso de trámites se convierten en artificios legales que distorsionan el mercado, excluyendo a los pequeños y medianos productores, pues los concentrados no se pueden importados directamente por ellos mismos. 

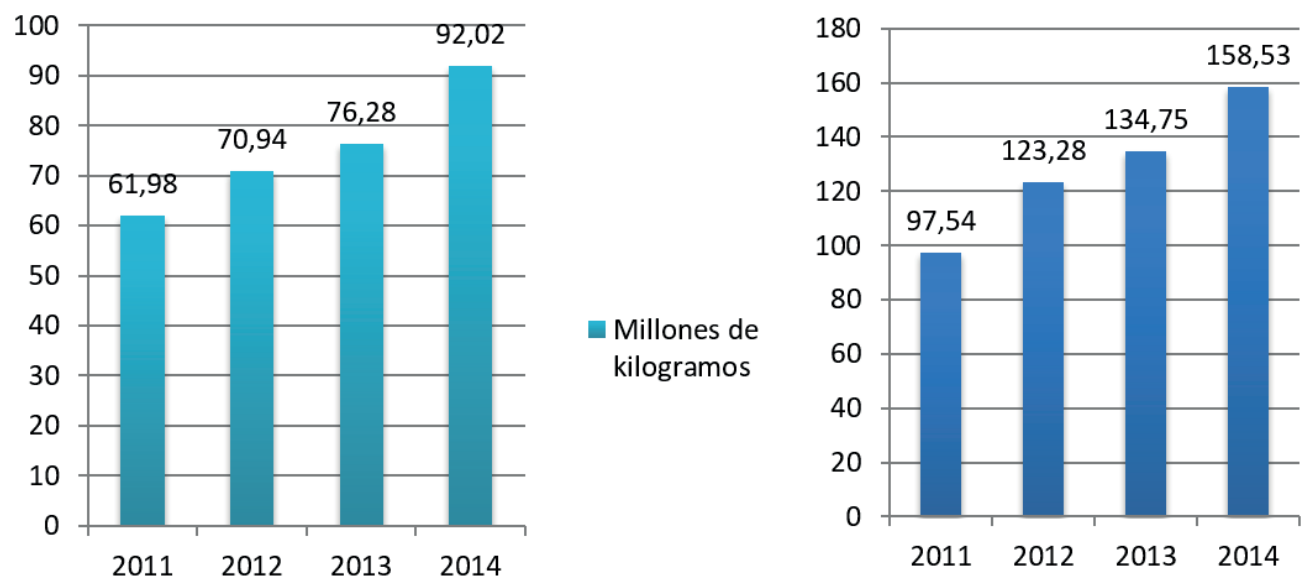

Millones de US\$

Gráfico 3. Costa Rica Volumen y valor de exportación del período 2011-2014 (millones de kilogramos y millones de US\$) Fuente: CNPL con datos de Ministerio de Hacienda (Tica), 2015.

Además, otros aspectos relevantes que deben tomarse en cuenta en relación con la cadena de valor es la presencia de intermediarios (servicios técnicos, distribución y consumo) que, de acuerdo con Ronald Torres , Administrador de la Asociación de Productores Agrícolas de Turrialba, recuperan una cantidad importante del valor agregado de los productos finales.

\section{Componente social de la actividad lechera}

Se estima que un 56\% de la Población Económicamente Activa (PEA) empleada en actividades agropecuarias no tiene secundaria completa (Gutiérrez, 2015). Según datos del Ministerio de Trabajo, el salario mínimo del sector agropecuario en Costa Rica es US\$ 640, incluidas las cargas sociales.

La producción de leche tiene efectos sociales importantes. En 2014, el 20.34\% que representó la mano de obra en la estructura porcentual de los costos de producción en fincas lecheras incluyó principalmente a trabajadores en lecherías, trabajadores del campo y pago de cargas sociales (Gráfico 4).

Otras estimaciones, realizadas en este caso por CNPL, señalan que el subsector lechero genera alrededor de 46.000 empleos entre fincas e industrias lácteas, lo que representaría el $5 \%$ del empleo del sector privado o el $17 \%$ de la población ocupada por el sector agropecuario (CNPL, 2015). 


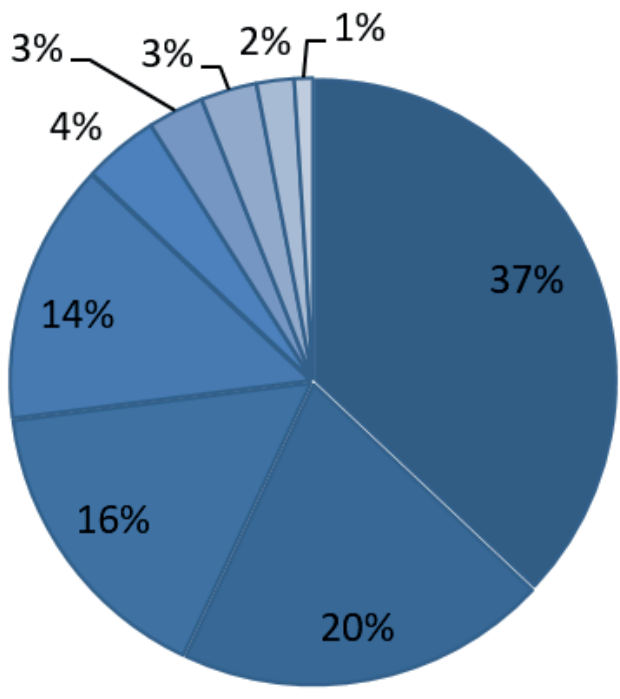

Lechería

Campo

Cargas sociales

Administrativos

Reemplazos

Servicios profesionales

Mantenimiento

Otros

Cultivos

Gráfico 4. Costa Rica. Distribución porcentual de la mano de obra en la estructura de costos de producción en fincas lecheras.

Fuente: Consultores en Agrogestión, S. A., 2015.

\section{Promoción de innovaciones institucionales dentro de la actividad lechera}

\subsection{Cambios históricos}

Diversos acontecimientos históricos han provocado cambios importantes en la dinámica productiva del subsector lechero. Como resultado de estas transformaciones, se ha propiciado, en mayor o menor medida, la incorporación de innovaciones, que surgen, en la mayoría de los casos, a partir de procesos de adaptación a las condiciones geográficas y climatológicas del país. Con la erupción del Volcán Irazú en el año 1963, la ganadería de altura comienza a descender pisos altitudinales, y a medida que esto ocurre, la actividad lechera cambia su sistema especializado.

Para Abarca (2015), empieza a verse el doble propósito como la opción más tradicional de bajura: un sistema de producción diferente y muy criollo, que no tiene similar en el resto del mundo. De acuerdo con los datos del Censo Agropecuario (Cenagro, 2014), el hato bovino en Costa Rica es de 1278.817 cabezas. De ese total, un 58\% (737.019 cabezas) corresponde a animales de los sistemas de producción de leche especializada y de doble propósito (CNPL, 2015). En el Gráfico 5 se muestra la distribución porcentual según el sistema de producción del que se trate 


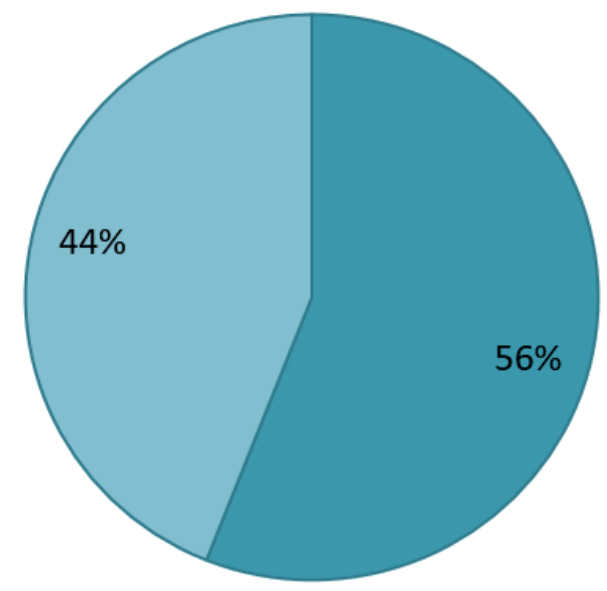

$\square$ Lechería especializada $\quad \square$ Lechería doble propósito

Gráfico 5. Costa Rica. Sistema de producción de leche en el 2014

Fuente: Elaboración propia con datos de Cenagro, 2014.

La pérdida de altitud, sin embargo, acarrea problemas de salud al ganado, que no logra adaptarse a los terrenos de bajura fácilmente. Cuando el productor moviliza las vacas hacia tierras más bajas, estas comienzan a enfermar y a presentar problemas reproductivos que antes no tenían, haciendo que el rendimiento de la actividad productiva decaiga. Los requerimientos nutricionales de esas vacas, además, no logran ser cubiertos con los pastos que se tienen en zonas bajas, motivando de esta manera que los productores utilicen su ingenio para identificar nuevos métodos de alimentación que garanticen la rentabilidad a pesar de las condiciones adversas. Este contexto en particular, aunado a las propuestas de apoyo hechas por institucionales del sector, ha dado forma a las estrategias de acción para la promoción de actividades dirigidas a fomentar procesos de innovación: genética, nutrición y manejo.

\subsection{Vinculación con el subsistema de innovación del subsector lechero}

Para Vargas, investigador de la Escuela de Medicina Veterinaria de la Universidad Nacional, el subsector lechero en Costa Rica cuenta con diversas iniciativas institucionales de calidad que, sin embargo, suelen aplicarse de forma aislada sin garantizar la inclusión de micro y pequeños productores. Gutiérrez (2015) plantea que es necesario un cambio de paradigma que permita la sostenibilidad económica y ambiental de la actividad lechera en el país, así como la participación activa de los productores en procesos de investigación. 
Abarca (2015) considera que no se ha promovido ninguna política pública para impulsar la competitividad del sector en los últimos 10 años, e incluso califica de "estancamiento" la situación actual del subsector, señalando que la poca vigilancia de las condiciones del entorno -nacional e internacional- y la falta de visión prospectiva del sector agropecuario costarricense funcionan como obstáculos a la innovación. De acuerdo con Óscar Solís , la escasez de líneas de crédito accesibles a pequeños productores, que permitan introducir mejoras de sus sistemas productivos, ha sido otro de los factores que impiden la innovación en el subsector.

Este panorama permite identificar la magnitud de los retos y las oportunidades del subsector lechero. Sin embargo, es importante destacar también acciones concretas que las organizaciones del sector han realizado para atender las necesidades durante el período correspondiente a la Administración Solís Rivera (Apéndice 2).

\subsection{El rol central del cooperativismo}

Las cooperativas tienen un rol central en la organización del subsector y la creación de competencias y capacidades de productores de leche. Según datos de la Cámara Nacional de Productores de Leche (2015), en 2012 el 88. 5\% de la leche se producía en fincas de productores asociados a cooperativas o miembros de asociaciones de productores.

Actualmente, la Cooperativa Dos Pinos, conformada por aproximadamente 1.400 socios, recoge alrededor del $80 \%$ de la leche que ingresa al circuito industrial. Para Sandra Zamora , Gerente de Innovación de esta empresa, uno de los principales beneficios de la relación cooperativa-productor es la mejora continua en las fincas, cuyos índices de productividad y calidad de la leche aumentan.

Héctor León considera que las Cooperativas distribuyen sus excedentes entre los productores asociados y esto les genera parte de la estabilidad en el negocio. Además, facilitan la prestación de servicios a través de la oferta de profesionales que emplean para dar soporte técnico al asociado. A pesar de ello, la mayoría de los pequeños y medianos productores de leche de bajura operan de forma independiente (Abarca, 2015), lo cual se convierte en un obstáculo al desarrollo inclusivo del subsector.

\subsection{Estrategias de acción}

La coordinación interinstitucional entre el Instituto Nacional de Innovación y Transferencia en Tecnología Agropecuaria (Inta) y la Cámara Nacional de Productores de Leche quedó patente durante la realización del Congreso Nacional Lechero 2015.

En esta actividad de notable relevancia para los productores de leche se discutió acerca de las estrategias de acción para la promoción de actividades dirigidas a promover procesos 
de innovación, dando seguimiento al Programa Pecuario, y en particular al Programa de Producción Animal, encargado de establecer los objetivos nacionales de innovación y transferencia tecnológica. Tal y como se muestra en la figura 1, las estrategias de acción se basan en la triada: génetica, nutrición y manejo.

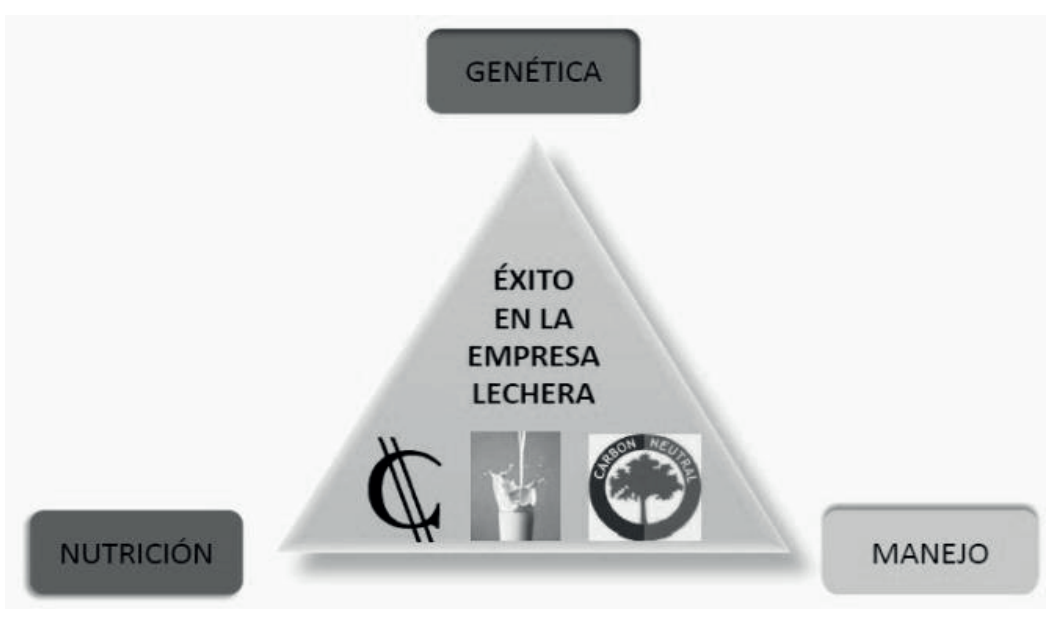

Figura 1. Costa Rica. Estrategias de acción para la promoción de actividades dirigidas a promover procesos de innovación

Fuente: León, 2015

\section{Mejoramiento genético}

El objetivo principal en genética es mejorar la salud del hato mediante la introducción de "toros resultantes de cruces con razas europeas lecheras (Simmental y Pardo Suizo)" (CNPL 2015, 34). En sus inicios, el Centro de Inseminación Artificial de El Alto, de Senasa, funcionó como receptor de toros nacidos en Costa Rica con semen importado, lo cual favoreció su distribución a precios bajos y el consecuente mejoramiento de la genética de la leche en Costa Rica. Sin embargo, este centro entró en declive, dejando de incidir en el mejoramiento genético, en especial de los pequeños productores. De acuerdo con Abarca (2015), actualmente solo los productores grandes son importadores directos de semen, siendo Canadá el principal origen de la importación (Cruz, 2015).

En la actualidad, la utilización de toros de monta continúa siendo una práctica frecuente entre los productores que operan bajo el sistema de doble propósito, especialmente aquellos que trabajan de forma independiente e informal. Por ende, se trabaja también en la identificación de un esquema organizacional para el fomento de la inseminación artificial, que permita al mismo tiempo su distribución entre los productores, en particular entre los pequeños y medianos; así como una estrategia de producción estacional que permita a los productores de leche dedicarse a otras actividades productivas durante la temporada lluviosa. Se trata, en todo caso, de acciones 
pendientes que requieren de la capacidad de extensión de las organizaciones del estado y la vinculación con el sector privado.

\section{Nutrición}

Costa Rica también es importador de genética para el mejoramiento de pastos y forrajes. El propósito general de esta estrategia es reducir la dependencia de granos, importados principalmente de Estados Unidos, y que se utilizan para fabricar el concentrado que alimenta a las vacas; de esta forma, se busca reducir costos de producción. El mejoramiento genético de pastos y forrajes además busca estimular su producción en zonas de bajura, donde la baja calidad de pastos locales afecta la productividad del ganado.

Los problemas asociados a la pérdida de escala de la producción de leche en el país, provocada en los últimos años por la tendencia al fraccionamiento de las fincas en unidades productivas más pequeñas, tuvo consecuencias negativas en el sistema de alimentación a base de pastoreo. Por ello, el sistema de alimentación está incorporando prácticas de rotación intensiva y semiconfinamiento (alimentos almacenados).

En esta línea, el proyecto NAMA Ganadería (Acciones Nacionalmente Apropiadas) está implementando acciones concretas como el desarrollo de sistemas de pastoreo más intensivos y suplementación con forrajes de corte y otros suplementos producidos por la propia finca. Si bien el ganado costarricense aún es altamente generador de $\mathrm{CO}_{2}$, las estrategias de acompañamiento al ganadero promueven una agricultura intensiva en conocimiento y más resiliente a los efectos del cambio climático.

En concreto, el NAMA Ganadería se enmarca dentro del conjunto de acciones país para la adaptación al cambio climático, y cuenta con la participación de 100 fincas, ubicadas en cinco zonas del país, que forman parte de la primera etapa del plan piloto. Se trata de un proceso de innovación institucional, enmarcado en la acción estratégica de las organizaciones del sector, que no solo busca orientar la ganadería de pequeña escala hacia una mayor productividad, sino que además se propone que la producción primaria alcance una mejor articulación con las industrias de carne y lácteos, generando así una mejor distribución del valor agregado obtenido por cada producto.

\section{Manejo}

De acuerdo con la Cámara Nacional de Productores de Leche (2015, p. 24):

El sistema ha evolucionado del pastoreo continuo al sistema de rotación intensivo para mejorar el manejo del pasto y de la vaca. En algunos casos, el pastoreo intensivo ha llevado a sistemas de semi-confinamiento donde se ofrecen alimentos almacenados para sostener la producción de leche cuando los forrajes no están disponibles o las pasturas requieren protegerse para no ser destruidas por el pisoteo de las vacas. 
En relación con la adaptación al cambio climático, la estrategia para los sistemas de manejo es la reducción de emisiones GEI y la fijación de $\mathrm{CO}_{2}$ (Inta, 2015). De acuerdo con Chacón (2015, comunicación personal), se trabaja actualmente en tecnológicas de adaptación a nivel de proceso (sistemas de pastoreo/silvopastoreo, sistemas de mejora genética, sistemas de manejo del agua) y de insumos (suplementación, fertilización, bancos forrajeros, variedades de pastos y leguminosas) lo que ha implicado para los productores la creación de invernaderos, la producción de biomasa y procesos de reconversión a la agricultura orgánica, entre otros procesos de cambio y mejora.

\section{Creación de competencias y capacidades}

A fin de comprender en detalle el tema de creación de competencias y capacidades, se sistematizaron las experiencias de tres organizaciones del sector lácteo costarricense: Cooperativa Dos Pinos R.L., integrada por más de 2000 asociados productores y trabajadores a lo largo del país; Cooperativa Agroindustrial de Servicios Múltiples de Puriscal R.L., ubicada en una zona de tradición rural dedicada principalmente a actividades agrícolas y ganaderas; y Asociación de Productores Agropecuarios de Santa Cruz (Asoproa), ubicada en Turrialba de Cartago, región conocida por ser el lugar de producción de quesos de alta calidad.

Estas organizaciones del sector, en conjunto con la Cámara Nacional de Productores de Leche y las industrias asociadas, participan de los esfuerzos por promover actividades de innovación. En relación con la producción primaria, las principales iniciativas para promover procesos de innovación se centran en la integración de los productores en la cadena productiva, la garantía de compra y comercialización de la leche producida y la reducción de oscilaciones abruptas en los precios (CNPL, 2015).

A nivel local, sin embargo, los productores a pequeña escala tienen necesidades particulares que requieren de políticas de innovación orientadas a la inclusión social. La alta demanda social de conocimiento en las zonas de bajura representa un desafío importante en términos de la orientación estratégica del conocimiento y su relación con la calidad de vida en los territorios con poco acceso al sistema formal de educación. En este apartado se analiza, mediante tres estudios de caso, las experiencias de productores asociados a cooperativas y miembros de asociaciones a partir del acceso y nivel de participación en actividades para la creación de competencias y capacidades.

\subsection{Cooperativa Dos Pinos R.L.}

Dos Pinos, la cooperativa de productores de leche más grande del país, pone a disposición de sus asociados un programa de transferencia tecnológica que, para la mayoría de servicios ofrecidos, no tiene costo alguno (Apéndice 3). Dos Pinos encabeza, además, la industria láctea de gran escala, liderando los procesos de calidad a través de la tecnificación y la modernización tecnológica. Cuenta en sus instalaciones con departamentos formales de innovación e 
investigación y desarrollo, enfocándose en dar un mayor valor agregado a la leche a través de productos diferenciados.

Los procesos de producción y calidad de Dos Pinos se han convertido en un referente de benchmarking para las industrias del subsector. Por ejemplo, Sigma Alimentos, que después de adquirir Monteverde y Lekerland, recibe la leche de aproximadamente 600 productores (CNPL, 2015), ha tratado de incorporar como parte de su estrategia servicios de veterinaria, agronomía y asistencia a la finca, similares a los implementados por Dos Pinos (Cruz, 2015; comunicación personal).

La ventaja competitiva de Dos Pinos sobre otras industrias del subsector se basa en el valor agregado del conocimiento de sus productores. Además de la distribución de sus excedentes entre los productores asociados, hecho que le genera al productor estabilidad en su actividad productiva, el programa de transferencia tecnológica de la Cooperativa mantiene una buena oferta de profesionales que trabajan de la mano con el productor (Hidalgo, 2015, comunicación personal), brindando capacitaciones en procesos básicos como el uso apropiado de ordeños mecánicos y los equipos de refrigeración, como tanques de enfriamiento, que se transfieren a la finca.

Este tipo de aprendizaje, definido por Lam (2002) como encoded knowledge se caracteriza por la sistematización de reglas y procedimientos, protocolos de trabajo que permiten la estandarización de los procesos, en especial el uso óptimo de los paquetes tecnológicos, sin que ello implique realmente la integración de productores a redes de trabajo o niveles altos de autonomía en la gestión de ideas.

\subsection{Cooperativa Agroindustrial de Servicios Múltiples de Puriscal R.L.}

Coopepuriscal R.L. representa un caso exitoso de vinculación de productores con organizaciones del subsistema de innovación del subsector lechero. En la zona de influencia de esta cooperativa, los productores de leche desarrollan el proyecto de construcción de la planta para el procesamiento y producción de lácteos. En la construcción de la planta, proyecto financiado por Infocoop, se ha vinculado el MAG y la Universidad de Costa Rica que, a través del Centro de Investigación en Economía Agrícola y Desarrollo Agroempresarial y el Trabajo Comunal Universitario TC-488 "Iniciativas comunitarias para el desarrollo rural de Puriscal, Acosta, Pejibaye", buscan mejorar la competitividad de la producción primaria de leche de la zona a través de una alternativa de cooperativismo.

El proyecto surge como alternativa a la ganadería de carne, previendo una caída posible de sus precios, y apoya, de esta manera, la actividad lechera, mediante el establecimiento de contratos que permitan definir cuotas específicas y precios, lo cual implica la formalización del sector en la zona y el mantenimiento de un ingreso constante para el productor. De acuerdo con Olga Calvo , en el proyecto participan el conjunto de productores de la zona de Puriscal, 61 en total, pese a que solamente 24 de ellos son asociados de Coopepuriscal R.L. Se espera que la planta de 
producción genere trabajo para 10 a 15 personas en la primera etapa y continúe generando empleo en las etapas posteriores.

Actualmente, los estudiantes de la Universidad de Costa Rica que realizan su TCU en la comunidad acompañan a los productores en la mejora de las unidades productivas. Algunas de las mejoras de proceso han sido el cementado y la capacitación en el uso a partir de la adquisición de tecnologías como tanques de enfrío. A nivel genético, se trabaja en la creación de competencias y capacidades para que los productores realicen la inseminación artificial (en la zona se sigue utilizando el toro para la monta, lo cual implica pérdida de eficiencia en la producción) y se brindan talleres participativos sobre la aplicación de semen sexado. También los jóvenes capacitan a los productores para que realicen cambios su sistema de producción (ordeño mecánico), apliquen la estandarización de procesos y metodologías para el registro de datos. Se trata de un tipo de aprendizaje basado en el conocimiento aplicado, learning-by-doing, que ha sacado provecho de la disposición a aprender de los productores locales, convirtiendo de esta forma las capacidades en habilidades prácticas para la mejora continua en operaciones fundamentales del proceso productivo.

La interacción entre diferentes actores del Sistema Nacional de Innovación ha hecho posible la introducción de cambios y mejoras en el proceso de producción. La innovación, que podría calificarse de tipo incremental, no solamente permite al productor el acceso a la información sino que le provee de una estructura de apoyo para la gestión de las prácticas introducidas en el marco de la vinculación.

Lo relevante de esta experiencia, sin embargo, son las características de las iniciativas institucionales; pues estas han identificado con claridad suficiente el perfil del productor de la zona y, en consecuencia, el tipo de actividades desarrolladas toma en cuenta el conocimiento tradicional presente en la zona, lo cual le permite al beneficiario un empoderamiento horizontal que garantiza una participación comprometida de todas las partes. Los nuevos conocimientos son incorporados de forma paulatina por los productores, pero son sostenibles en el tiempo, dado que la absorción de la tecnología se acompaña, si bien en un nivel básico, de la gestión de aprendizaje.

\subsection{Asociación de Productores Agropecuarios de Santa Cruz (Asoproa)}

Asoproa se ubica en Santa Cruz de Turrialba, una zona de tradición ganadera, cuya producción artesanal de leche se dedica en su mayoría a la fabricación de quesos frescos reconocidos incluso a nivel internacional. Asoproa, que cuenta actualmente con 250 productores asociados, se establece a causa de un problema climático en el año 2000, representó un proyecto de organización para solicitar ayuda al Gobierno y mejorar los forrajes y las condiciones estructurales de las fincas.

Desde entonces, esta asociación ha funcionado como plataforma de servicios y medio 
para capacitar a los productores. Las mejoras más importantes se han originado a partir de la toma de conciencia ambiental de los productores, motivando cambios en el proceso productivo: disminución de la recarga animal por hectárea, siembra de forrajes, invernaderos y biodigestores para convertir los desechos en abono orgánico. De acuerdo con Torres (2015), en los últimos años una cantidad considerable de productores de la zona ha dejado de ordeñar a mano para tecnificar el proceso. La misma situación ocurre con la monta de la vaca, pues la inseminación artificial se ha convertido en una práctica más frecuente.

En la comercialización, se lograron alianzas para colocar el queso producido en ferias del agricultor, mediante un acuerdo de colaboración alcanzado con el Comité de Ferias de la Zona Oriental. La meta de Asoproa es aumentar el precio del queso. Torres (2015) reconoce que no se ha sacado provecho de la Denominación de Origen que tiene el Queso Turrialba, cuya explotación podría ayudarlos a lograr ese objetivo. Además, los productores de esta organización consideran que la competencia, en particular Dos Pinos y Sigma Alimentos, mantiene ventajas competitivas importantes a nivel del posicionamiento en el mercado nacional, a través de redes de suministro y comercialización consolidadas y estrategias de marketing y publicidad de productos de amplia cobertura en medios de comunicación y redes sociales. La capacidad productiva de ambas organizaciones, además, es central para que estas continúen regulando los precios de los lácteos en épocas de sobreproducción.

En Asoproa, la producción se organiza de forma individual (a lo interno de cada núcleo familiar) y no existen canales de colaboración entre productores . Esto representa un obstáculo a la gestión de las ideas y la innovación, puesto que los flujos de información que podrían derivar en insumos para la mejora en la finca o el proceso productivo no circulan entre productores ni la organización. No existe suficiente cohesión social en la zona que facilite una organización del trabajo efectiva ni la implementación de acciones colectivas que garanticen el fortalecimiento de una estructura de apoyo, incluso como medio de acceso a la interacción con instituciones públicas.

La creación de capacidades, por lo tanto, resulta disminuida por la debilidad institucional, incluyendo la ausencia de una visión de desarrollo local compartida. Sin embargo, los procesos de adaptación que han acompañado la evolución de esta organización pueden considerarse como un incentivo necesario para el estímulo de eco-innovaciones.

\section{Implicaciones de política: áreas estratégicas de acción}

El subsector lechero requiere de la reactivación de una política pública integral que fomente la competitividad en áreas estratégicas de la producción y la comercialización de leche y derivados. La situación actual del subsector lechero advierte la necesidad de una intervención institucional creativa que permita, mediante la implementación de acciones en el corto plazo, brindar respuestas sostenibles a los problemas derivados de la creciente inestabilidad del 
contexto macroeconómico internacional y de la fuerte dependencia de los mercados externos. En el plano económico, los precios de los productos lácteos muestran una tendencia general a la baja y se espera el aumento de la carga impositiva y las tasas de interés, lo cual tendrá consecuencias directas sobre la dinámica de inversión del subsector y el empleo.

Una mayor -y mejor contralada- supervisión del entorno productivo puede permitir la identificación de oportunidades de maniobra para la industria láctea y los productores, en particular los pequeños y medianos. La promoción de iniciativas de ecoeficiencia en el manejo de fincas lecheras resulta un instrumento clave para reducir los costos de producción y contribuir a la protección ambiental del planeta. El Estado costarricense ha dado pasos importantes al impulsar proyectos como el NAMA Ganadería y la Estrategia Nacional de Ganadería Baja en Carbono; sin embargo, el éxito de estas acciones dependerá del nivel de compromiso y la participación activa de todos los actores involucrados.

Es urgente establecer además vinculaciones público-privadas que permitan mejorar los términos de intercambio y el posicionamiento de los productores de leche dentro de la cadena de valor de los productos lácteos. Asimismo, resulta evidente la necesidad de dotar con recursos financieros los canales de financiamiento que hacen posible la apertura y mantenimiento de programas de investigación y transferencia tecnológica. A nivel sectorial, el Ministerio de Agricultura y Ganadería cumple un rol protagónico en la definición de políticas dirigidas al subsector, una función que debe cumplir en estrecha colaboración con el conjunto de la institucionalidad pública y otras organizaciones privadas, como la Cámara Nacional de Productores de Leche, que recientemente reconoció la importancia de consolidar plataformas de coordinación interinstitucional y el establecimiento de una agenda común en materia de capacidad técnica y creación de capacidades.

Finalmente, las organizaciones del subsector lechero deben evaluar los efectos de su actividad en el desarrollo social. Los costos de los servicios públicos, la disposición y estado de infraestructura para la producción y la relación desigual en los términos de intercambio son tres de los principales desafíos en materia de política económica.

\section{Conclusiones}

Se distingue la importancia que el subsector lechero mantiene dentro de la actividad agropecuaria costarricense. Pese a los obstáculos a la producción primaria de leche identificados en este estudio, el escenario permite determinar elementos clave que, de ser tomados en cuenta, pueden mejoran el desempeño económico y la competitividad de los actores directos.

Considerando el concepto de Sistema Nacional de Innovación, las organizaciones del subsector lechero tienen un rol importante en la definición de las estrategias de acción y actividades dirigidas a promover procesos de innovación, especialmente a través de la adquisición de tecnología y la capacitación a productores. Sin embargo, existen reglas del juego establecidas 
que afectan la inclusión social de micro y pequeños productores en segmentos específicos de la cadena de valor, como la comercialización.

El subsector lechero se ha visto afectado en los últimos años por la dependencia de granos, materia prima de los concentrados que consumen las vacas. A nivel nacional, una cantidad reducida de proveedores controla ese mercado a través de la importación directa y la fijación de los precios. Por tal razón, la Cámara Nacional de Productores de leche, en conjunto con instituciones del Estado, ha comenzado a promover recientemente la producción de forrajes con mayor carga nutricional y alimenticia para animales en zonas de bajura. Sin embargo, el éxito de esta propuesta dependerá del reconocimiento de los efectos del cambio climático sobre los sistemas socioecológicos locales y la interacción de los productores con el paisaje.

En relación con el entorno productivo del subsector, la Cooperativa Dos Pinos continúa liderando los procesos de innovación e I+D, así como los estándares de calidad y el establecimiento de precios. Por ende, el desarrollo inclusivo del sector dependerá de la apertura institucional a trabajadores independientes y la colaboración constante con cooperativas y asociaciones.

La transferencia tecnológica en el subsector, pese a su importancia en términos de mejoramiento genético del ganado y los pastos, no garantiza la inclusión de pequeños productores. Los altos costos y deficiencias en el manejo del proceso productivo, así como la poca visibilización de factores socioculturales constituyen frenos a la participación inclusiva y el acceso a nichos de mercados sostenibles. Conceptos operativos como aprendizaje social y resiliencia pueden contribuir a la identificación de estrategias transdisciplinares de desarrollo, considerando que la disposición al uso de tecnología forma parte de un proceso más amplio de transformación cultural e histórica.

La gestión de la innovación en el subsector se basa en la transferencia tecnológica. De esta manera, se desaprovecha el conocimiento tácito del productor, en especial de aquellos con edad avanzada, que podría estimular los procesos innovación y creatividad, apalancando nuevas perspectivas para el fortalecimiento de "comunidades de prácticas" en el subsector. Aunado ello, se debe promover el relevo generacional dentro del subsector a través de actividades de promoción para interesar a los descendientes de productores a seguir con la producción de leche, como lo ha hecho Dos Pinos R. L. Esta estrategia, sin embargo, debe considerar las inequidades estructurales de acceso a educación, salud y protección del ambiente que funcionan como causales de exclusión para la población joven de zonas con menor desarrollo.

Se plantea un escenario complejo a partir de la desregulación arancelaria que en vigencia desde el año 2016. Sin embargo, se trata también de un escenario oportuno para la mejora inclusiva del sector, lo que supone una mayor participación de los productores a pequeña escala en los procesos de transferencia tecnológica, así como un mejor aprovechamiento de su experiencia empírica en el manejo del proceso productivo que, de modo paralelo, contribuirá al fortalecimiento de capacidades endógenas para el desarrollo local. 
Revista de Política Económica • EISSN: 2215-4167

Vol. 2 (1) • Julio-Enero, 2016: 1-25

DOI: http://dx.doi.org/10.15359/peds.2-1.2

Barboza-Arias

URL: http://www.revistas.una.ac.cr/politicaeconomica

\section{Bibliografía referenciada}

Arocena, R. \& Sutz, J. (2002). Sistemas de Innovación y Países en Desarrollo. Universidad de la República de Uruguay. Recuperado de: http://portales.puj.edu.co/jaguilar/Paisesdesarrollo.pdf

CINPE. (2015). Manual para la gestión de procesos de innovación. Documento de trabajo. UNA, Heredia, Costa Rica. Recuperado de: http://repositorio.una.ac.cr/bitstream/ handle/11056/11477/MANUAL\%20VERSION\%20COMPLETA\%20\%281\%29. pdf? sequence $=1 \&$ is Allowed $=y$

Cámara Nacional de Productores de Leche [CNPL]. (2015). Revista Horizonte Lechero, Setiembre (2). Recuperado de: https://issuu.com/proleche/docs/revista horizonte lechero setiembre

Instituto Nacional de Estadísticas y Censes. (2014). VI Censo Nacional Agropecuario.. Recuperado de: http://www.inec.go.cr/censos/censo-agropecuario-2014

Edquist, C. (ed.). (1997). Systems of Innovations: Technologies, Institutions and Organizations. Wiltshire, Great Britain: John de la Mothe, Series Editor. Retrieved from: https:// charlesedquist.files.wordpress.com/2015/06/science-technology-and-the-internationalpolitical-economy-series-charles-edquist-systems-of-innovation -technologiesinstitutions-and-organizations-routledge-1997.pdf

Gutiérrez, L. (octubre de 2015). Análisis de la competitividad del sector lácteo costarricense: Visión de la Cámara Nacional de Productores de Leche. Ponencia presentada en el Congreso Lechero 2015. San José, Costa Rica. Recuperado de: http://proleche.com/ recursos/documentos/congreso2015/produccion/Charla1.pdf

Lam, A. (1998). Tacit knowledge, organizational learning and innovation: a societal perspective. DRUID working paper $N^{\circ}$ 98-22. Retrieved from: https://pdfs.semanticscholar.org/707c/ af11b2eb0fb931e76a412bd221166420db28.pdf

Lam, A. (2002). Alternative Societal Models of Learning and Innovation in the Knowledge Economy. DRUID. Working paper. Retrieved from: https://pdfs.semanticscholar. org/0ce4/8550d64c0c56a07faf872865571d4985ed45.pdf

León, H. (octubre de 2015). Eficiencia en el costo de alimentación y la mano de obra. Ponencia presentada en el Congreso Lechero 2015. San José, Costa Rica. Recuperado de: https:/ganaderiasos.com/wp-content/uploads/2017/09/EFICIENCIA-EN-LA-EMPRESALECHERA-EL-COSTO-DE-PRODUCCI\%C3\%93N.pdf

Lundvall, B. (1992). National Systems of Innovation. Towards a Theory of Innovation and Interective Learning. London: Printer Publishers. 
MAG. (2015).Informe técnico de resultados del $1^{\circ}$ año de Gobierno. Costa Rica. Recuperado de:http:// www.infoagro.go.cr/MarcoInstitucional/Documents/INFORMES/Informe 0105 2015SECTORAGRO.pdf

Orozco, J.; Ruiz, R.; y Corrales, R. (2015). Manual para la Gestión de la Innovación. http:// www.repositorio.una.ac.cr/bitstream/handle/11056/11477/MANUAL\%20VERSION\%20 COMPLETA\%20\%281\%29.pdf?sequence $=1 \&$ isAllowed $=\mathrm{y}$

SEPSA. (2015). Informe técnico de resultados del $1^{\circ}$ año de Gobierno Mayo 2014 - Abril 2015. Costa Rica: MAG. Recuperado de: http://www.infoagro.go.cr/MarcoInstitucional/Documents/ INFORMES/Informe 0105 2015-SECTORAGRO.pdf

van Dijk, M. P. \& Sandee, H. (2002). Innovation and Small Enterprises in the Third World. United Kingdom: Edward Elgar, Cheltenham. Doi: https://doi.org/10.4337/9781781951323

Yin, R.(1994).CaseStudyResearch.Designand Methods. London:Sage Publications. Retrievedfrom: http://www.madeira-edu.pt/LinkClick.aspx?fileticket=Fgm4GJWVTRs\%3D\&tabid=3004

\section{Páginas web consultadas:}

Cámara Nacional de Productores de Leche. (2015). Memorias del Congreso Nacional Lechero 2015. Recuperado de: http://proleche.com/index.php/component/content/article?id=125

Cooperativa Dos Pinos R.L.: http://www.dospinos.com/app/cms/www/index.php

Instituto Nacional de Innovación y Transferencia en Tecnología Agropecuaria (INTA): http:// www.inta.go.crl

Ministerio de Agricultura y Ganadería (MAG): http://www.mag.go.cr/

Ministerio de Hacienda: http://www.hacienda.go.cr/

Sistema de Información del Sector Agropecuario Costarricense (Infoagro): http://www.infoagro.com/ 
Revista de Política Económica • EISSN: 2215-4167

Vol. 2 (1) • Julio-Enero, 2016: 1-25

DOI: http://dx.doi.org/10.15359/peds.2-1.2

Barboza-Arias

URL: http://www.revistas.una.ac.cr/politicaeconomica

\section{APÉNDICE 1.}

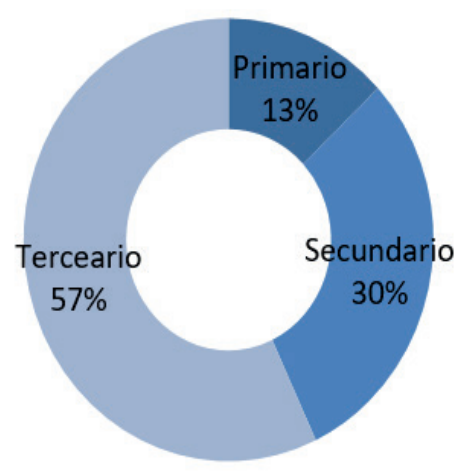

1991

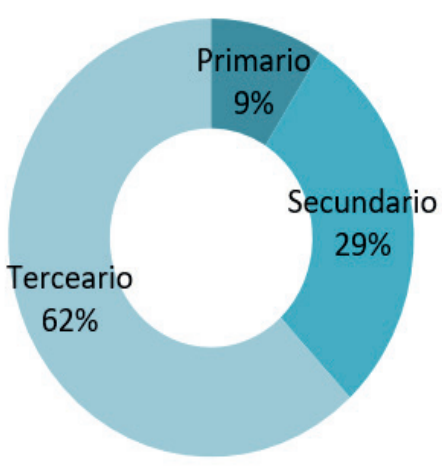

2014

Costa Rica: Estructura del Producto Interno Bruto por Sectores Productivos, 1991 y 2014

Fuente: Documento de trabajo CINPE, 2015. 
Revista de Política Económica • EISSN: 2215-4167

Vol. 2 (1) • Julio-Enero, 2016: 1-25

DOI: http://dx.doi.org/10.15359/peds.2-1.2

Barboza-Arias

URL: http://www.revistas.una.ac.cr/politicaeconomica

\section{APÉNDICE 2}

Acciones institucionales y gestión de iniciativas para la innovación en el subsector lechero, 2014-2015.

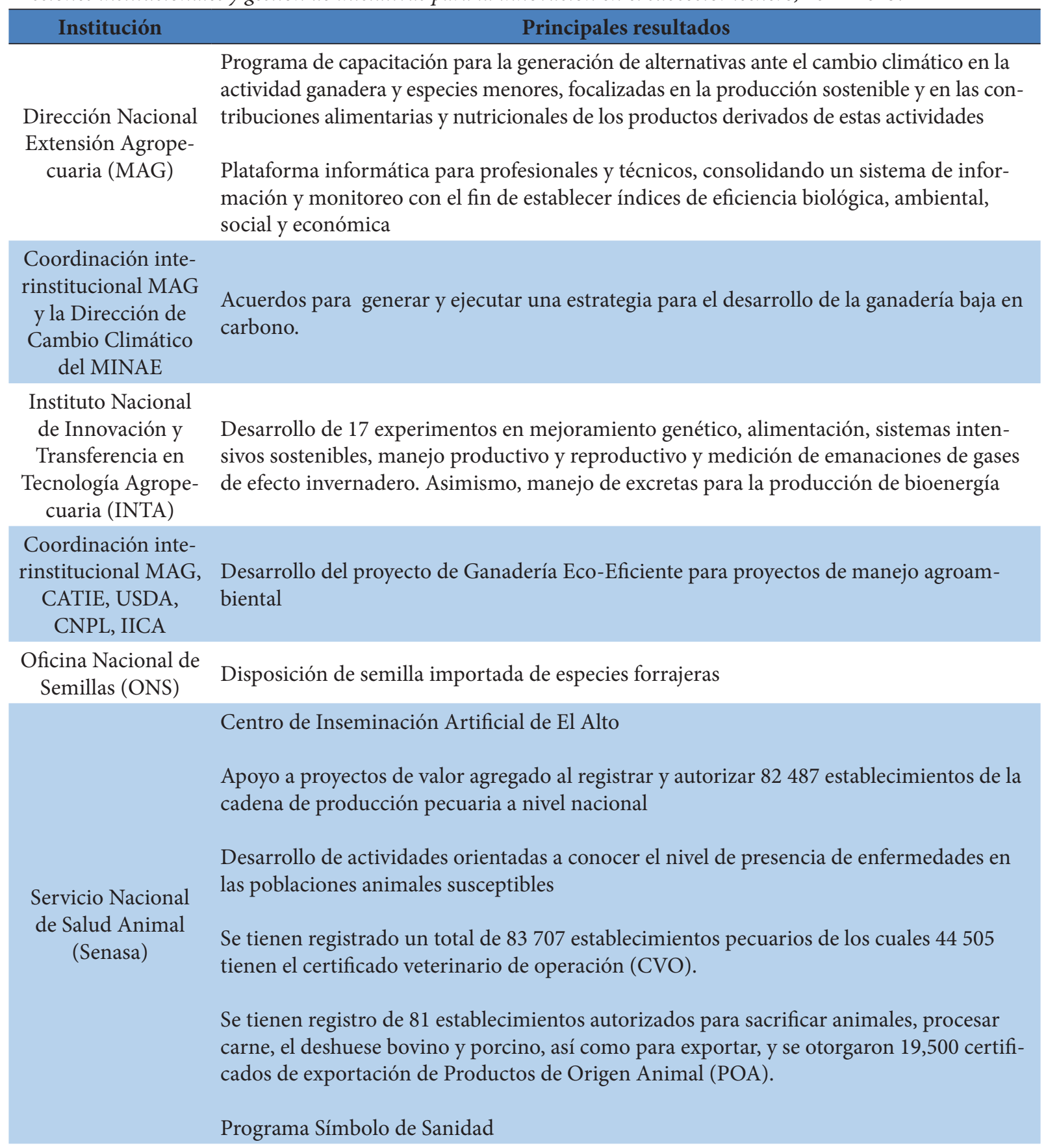


Revista de Política Económica • EISSN: 2215-4167

Vol. 2 (1) • Julio-Enero, 2016: 1-25

DOI: http://dx.doi.org/10.15359/peds.2-1.2

Barboza-Arias

URL: http://www.revistas.una.ac.cr/politicaeconomica

Servicio Fitosanita- Establecimiento de nuevas Medidas Sanitarias y Fitosanitarias, necesarias para proteger la rio del Estado (SFE)

Cámara Nacional de Productores de Leche vida y la salud de las personas y los animales, y para preservar los vegetales Propuesta de apoyo al MAG para la creación de una Red de Investigación en Forrajes y otros alimentos para Ganado Lechero

Alianzas estratégicos con MAG para los efectos del fenómeno meteorológico El Niño

Instituto Nacional Programas de mejora de capacidades para la producción de valor agregado con productode Aprendizaje res de leche a nivel nacional

\begin{tabular}{|c|c|}
\hline \multirow{3}{*}{$\begin{array}{l}\text { Universidad } \mathrm{Na}- \\
\text { cional }\end{array}$} & Capacitaciones continuas \\
\hline & Software VAMPP \\
\hline & $\begin{array}{l}\text { Apoyo en salud y reproducción a través de la Escuela de Medicina Veterinaria y su gama } \\
\text { de análisis de laboratorio }\end{array}$ \\
\hline \multirow{4}{*}{$\begin{array}{l}\text { Universidad de Cos- } \\
\text { ta Rica }\end{array}$} & Investigaciones en nutrición y alimentación, crianza de reemplazo \\
\hline & Laboratorio de calidad de alimentos CINA y de suelos CIA \\
\hline & Estación Experimental Alfredo Volio Mata \\
\hline & Trabajos Comunales Universitarios \\
\hline \multirow{3}{*}{$\begin{array}{l}\text { Universidad Técnica } \\
\text { Nacional }\end{array}$} & Investigación de ganadería de bajura (doble propósito) \\
\hline & \\
\hline & $\begin{array}{l}\text { Convenios con CIAT pata investigar opciones forrajeras como el Arachis pintoi, Madero } \\
\text { Negro y otros }\end{array}$ \\
\hline
\end{tabular}

Fuente: Elaboración propia con datos de CNPL, 2015; SEPSA, 2015 y entrevistas personales realizadas a Abarca (2015), Vargas (2015), Solís (2015) y Calvo (2015). 
Revista de Política Económica • EISSN: 2215-4167

Vol. 2 (1) • Julio-Enero, 2016: 1-25

DOI: http://dx.doi.org/10.15359/peds.2-1.2

Barboza-Arias

URL: http://www.revistas.una.ac.cr/politicaeconomica

\section{APÉNDICE 3}

Programa de Transferencia Tecnológica de la Cooperativa Dos Pinos R.L.

$\begin{array}{ll}\text { Servicio } & \text { Descripción } \\ & \end{array}$

Programa de Fertilización (PF) Toma de muestras y análisis de información para la elaboración del Programa de Fertilización, al cual se le brinda seguimiento en conjunto con el asociado productor.

Balance Nutricional de vacas en Toma de muestras de insumos de alimentación y análisis de datos para la elaproducción $(\mathrm{BN}) \quad$ boración del Balance Nutricional de vacas en producción, al cual se le brindará seguimiento en conjunto con el asociado productor.

Crianza de Reemplazos (CR) Análisis general del programa de crianza de reemplazos de la finca. Se brindará las recomendaciones en la mejora de la crianza en conjunto con el asociado productor.

Manejo de vacas secas (MVS) Análisis general del manejo de vacas secas; las recomendaciones de mejora se brindarán en conjunto con el seguimiento brindado al productor asociado.

Eventos de capacitación para

Capacitaciones grupales a nivel regional para transferir conocimientos y habiasociados y ganaderos lidades al asociado productor y su personal laboral.

Reproducción y Salud (RS) Análisis general de la reproducción y salud del hato: las recomendaciones de mejora se brindarán en conjunto con el seguimiento brindado al productor asociado.

Manejo de Forrajes (MF) Evaluación del manejo de forrajes utilizado en finca: siembras de pasto, variedades de pasto utilizadas, control de malezas, disponibilidades de pasto, carga animal, período de rotación, tamaño de aparatos, programas de fertilización, utilización de enmiendas, conservación de forrajes, análisis bromatológico. Recomendaciones y seguimiento al programa de manejo de forrajes.

Planificación y Gestión (PG) El asociado recibe apoyo para formar habilidades gerenciales en la finca: desde la planificación anual y definición de metas hasta estudios básicos de viabilidad para la valoración de nuevas inversiones.

Apoyo informático (VAMPP) El servicio de apoyo informático se basa fundamentalmente en el monitoreo del manejo del hato a través del software VAMPP, así como el monitoreo de los costos de producción con el software Dairy Profit.

Programa Agroambiental Se ofrece asesoramiento en el manejo y aprovechamiento del recurso boñiga de las fincas. Se diseña y asesora en la implementación del sistema a construir. Adicionalmente, se asesoraría a los asociados en la implementación de sistemas agroforestales, así como en el manejo de desechos sólidos ordinarios (envases plásticos). 
Revista de Política Económica • EISSN: 2215-4167

Vol. 2 (1) • Julio-Enero, 2016: 1-25

DOI: http://dx.doi.org/10.15359/peds.2-1.2

Barboza-Arias

URL: http://www.revistas.una.ac.cr/politicaeconomica

Asesoría en Costeo Lechero

(ACL)

Es una alternativa de apoyo técnico que tiene como objetivo mejorar la labor gerencial del asociado productor en su empresa lechera. Esto se obtiene mediante la inclusión mensual de los ingresos y egresos en un sistema computarizado, generando un estado de resultados y un reporte de bioindicadores diseñados específicamente para una toma de decisiones.

Regencia Almacén Agroveterina- Capacitación del personal responsable de la venta y prescripción de productos rio (REG) veterinarios y agrícolas, así como de la atención de consultas en las mismas áreas. Atención de emergencias según disponibilidad de tiempo.

Servicio

Descripción

Documentos de apoyo

Elaboración de artículos técnicos para transferir conocimientos a los asociados productores y ganaderos. La agenda de la finca es una herramienta de trabajo que tiene como objetivo aumentar la recopilación de información de los principales eventos productivos y reproductivos.

Determinación, rediseño y medi- Georeferenciación de las áreas de la finca, numeración y medición de aparatos, ción de áreas (GPS) distribución homogénea de los mismos, planes de fertilización de precisión, numeración.

Salud del hato

Dar apoyo a nivel de fincas para la salud en general del hato (sobre mastitis, hemoparásitos, problemas metabólicos, renqueras, indigestiones, problemas respiratorios, uso de medicamentos etc.).

Programa Tuberculosis y Brucelosis $^{*}$

- Revalidar certificados de hato libre o sanear fincas infectadas (Brucelosis y Tuberculosis). - En caso de sospecha de tuberculosis, se realiza la aplicación cervical comparativa. - Vacunación de terneras contra brucelosis. - Marcaje de animales positivos y emisión de guías sanitarias para transporte a matadero. Establecimiento de medidas tales como períodos de cuarentena y saneamiento de hatos infectados. - Análisis epidemiológico de fincas cercanas a hatos infectados. - Trámite para obtención de certificados de hato libre por parte de SENASA

${ }^{\star}$ El Programa Tuberculosis y Brucelosis es el único servicio ofrecido por Dos Pinos a sus asociados que tiene costo.

Fuente: Elaboración propia con datos de Cooperativa Dos Pinos R.L., 2015. 